\title{
EDITORIAL
}

For reprint orders, please contact: reprints@futuremedicine.com

\section{Is pediatric Clostridium difficile infection associated with prior antibiotic exposure?}
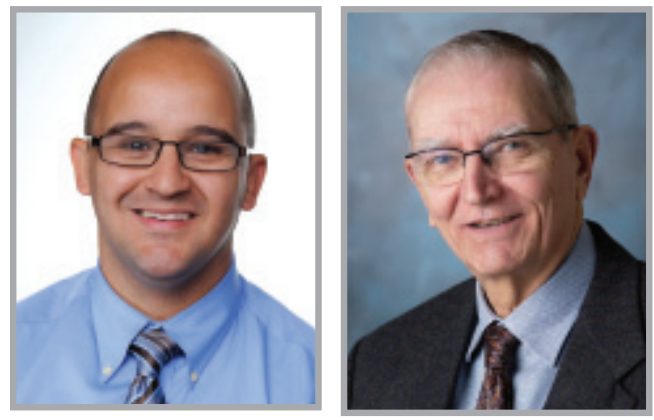

“...pediatric Clostridium difficile

infection is reported

increasingly among children

without hospital or antibiotic

exposure.”

Larry K Kociolek ${ }^{*, 1,2}$ \& Dale N Gerding ${ }^{3,4}$

Initially discovered by Hall and O'Toole in 1935 as a stool commensal in a healthy neonate [1], Clostridium difficile was first reported as the pathogen responsible for antibiotic-associated pseudomembranous colitis in 1978 [2]. Since then, C. difficile infection (CDI) pathogenesis has been well described $[1,3]$. During hospitalization and exposure to $C$. difficile spores, two factors contribute to developing CDI: disturbances of the healthy, highly variable colonic microbiota and lack of preformed $C$. difficile antitoxin antibodies and/or failure to develop an adequate humoral immune response to $C$. difficile toxins. For these reasons, many novel therapies for CDI have emerged, including microbiota-sparing antibiotics, probiotics/biotherapeutics (e.g., nontoxigenic strains of $C$. difficile and fecal microbiota transplantation) and immune-enhancing approaches such as $C$. difficile monoclonal antitoxin antibodies and vaccines [4].

The association of CDI and recent use of clindamycin or $\beta$-lactam antibiotics is well known. The dramatic rise in the incidence and severity of CDI among adults in the early part of the last decade was related to widespread fluoroquinolone use coincident with the emergence and spread of a novel highly fluoroquinolone-resistant strain of $C$. difficile (i.e., BI/NAP1/027) [1]. For these reasons, the CDC classifies $C$. difficile as one of three antibioticresistant infections that "are immediate public health threats that require urgent and aggressive action" [5]. Thus, antibiotic stewardship programs are essential to reduce CDI incidence [6]. However, $\mathrm{CDI}$ is also rising among patients at relatively low risk of infection, such as healthy children without recent hospitalization or antibiotic exposure [1-3]. Although recent antibiotic use is reported in $>85 \%$ of adult patients with CDI, recent antibiotic exposure is less frequently reported ( $35-75 \%)$ among children with CDI [3]. Multiple confounders, including unnecessary testing of children who are more likely to be colonized than infected with $C$. difficile, may be responsible for the apparent lower exposure to antimicrobials in children.

\section{KEYWORDS}

- antibiotics • Clostridium difficile - epidemiology $\bullet$ pediatric $\bullet$ risk factors

\section{"...Clostridium difficile was first reported as the pathogen responsible for antibiotic-associated pseudomembranous colitis in 1978."}

Future 


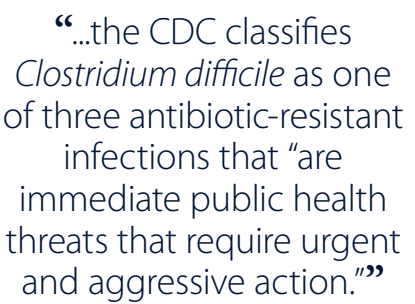

and aggressive action."”
In an observational, retrospective cohort study, Benson and colleagues described CDI among 513 children over a 5-year period [7]. Most children in this study had community-associated (CA) $\mathrm{CDI}$, and the proportion with CA-CDI increased over time. Recent antibiotic exposure (undefined as to time period before CDI), determined by medical record review, was reported among only $57 \%$ of patients. However, $39 \%$ of patients in this study were under 2 years old (an age when asymptomatic $C$. difficile colonization is frequent and unrelated to antibiotic exposure), and patients as young as 2 weeks old were included. In contrast, Sandora and coworkers described the epidemiology of pediatric CDI in a nested case-control study [8]. Ninety-five children diagnosed with CDI over an 8-month period were compared with 238 unmatched controls, and infants under 12 months old and patients without diarrhea were excluded to avoid confounding by inclusion of likely asymptomatic $C$. difficile carriers. Among children with CDI, $75 \%$ had received an antibiotic in the previous 4 weeks. Receipt of fluoroquinolone (odds ratio [OR]: 17.04; 95\% CI: 5.86-49.54) and nonquinolone (OR: 2.23 ; 95\% CI: 1.18-4.20) antibiotics in the previous month was associated with CDI.

Multiple recent studies describe risk factors and outcomes related to specific subgroups of pediatric CDI, such as severe and CA-CDI. Kim and colleagues compared 48 hospitalized children with severe CDI to 34 children with nonsevere CDI over a 21-month period in a nested case-control study [9]. Medical records and patient family interviews provided antecedent exposure data. Only receipt of at least three different antibiotic classes in the previous month was associated with severe CDI (adjusted OR: 3.95; 95\% CI: 1.19-13.11). However, the severe CDI group included a significantly greater proportion of children with malignancy or cystic fibrosis. Because these conditions are associated with increased antibiotic use, patient comorbidities may have confounded this association. Tschudin-Sutter and coworkers compared 38 inpatients with CA-CDI to 144 inpatients with healthcare facility associated CDI over a 9-year period in a retrospective, observational cohort study [10]. Antibiotic exposures in the previous 30 days were reported in $77 \%$ of children with healthcare facility-associated CDI but only $42 \%$ of children with CA-CDI ( $p=0.011)$, a difference potentially attributable to ascertainment of exposures only from patient medical records.
Each study described above was limited to a single medical center. However, Wendt and colleagues described 944 CDI cases identified through active CDI surveillance across ten diverse US geographic locations [11]. Children 1-17 years old who tested positive for C. difficile were included regardless of symptoms. Medication exposures in the previous 14 days were collected from medical records for all cases, and additional medication exposures within the previous 12 weeks were collected by interview of 95 patients with CA-CDI. Antibiotic use in the previous 2 weeks, which was similar across all age groups, was documented in $33 \%$ of patients. Among the 95 patients interviewed, $73 \%$ reported antibiotic use in the previous 12 weeks, demonstrating the limitation of obtaining antibiotic exposure from medical records alone.

These data suggest that pediatric CDI may not be closely associated with recent hospitalization and antibiotic exposure, both of which are well-described adult CDI risk factors. Whereas noninpatient healthcare facility exposures are common among adults with CA-CDI [12], this has not been studied in children. However, exposure to $C$. difficile spores in of itself is likely insufficient to cause CDI in the presence of a mature intestinal microbiome, particularly in those with preformed $C$. difficile antitoxin antibodies. Unlike in adults [13], the pediatric host immune response to $C$. difficile exposure has not been well described and warrants further investigation.

For many reasons, the reliability of published data describing pediatric CDI risk factors and outcomes may require careful re-examination. Misclassification and ascertainment biases may be particularly common in pediatric studies because of frequent $C$. difficile colonization among young children and patients of all ages with recent healthcare facility exposures $[2,3]$. Numerous case-control studies have reported similar prevalence of $C$. diffcile positive stools among children irrespective of CDI symptoms [14]. At our institution, a substantial proportion of patients with community-onset diarrhea who test positive for $C$. difficile have symptoms and exposures that are more consistent with a viral etiology, and few undergo additional testing for gastrointestinal viruses [15]. Therefore, pediatric studies may misclassify many patients as having CDI when they are more likely asymptomatic C. difficile carriers with another cause of diarrhea. Infants under 1-2 years of age are unique 
because of apparent resistance to symptomatic infection despite high prevalence of $C$. difficile colonization. The reasons for infant resistance to symptomatic CDI are not entirely clear, but lack of expression of receptors for toxin A by infant enterocytes is a leading theory [3]. Therefore, risk factor and outcome analyses in studies that include infants are biased because of inclusion of a relatively higher proportion of asymptomatic C. difficile carriers.

Published studies describing pediatric CDI vary significantly in design (i.e., retrospective vs. prospective) and data sources (e.g., administrative databases, hospital medical records and/or patient interviews), potentially leading to under-reporting of antibiotic exposures from incomplete documentation in the medical record and/or recall bias during patient interviews. Inpatient antibiotic exposures preceding healthcare facility-associated CDI are likely to be captured reliably because of accurate documentation in the medical administration record. However, reliable capture of antibiotic exposures preceding CA-CDI requires accurate patient recall and healthcare provider documentation. Therefore, pediatric studies, which often include a substantial proportion of patients with CA-CDI, may underestimate the association between pediatric $\mathrm{CDI}$ and antibiotics.

Antibiotic exposure definitions vary among pediatric CDI studies, ranging from 14 to 90 days prior to onset of CDI. Although CDI risk is highest 2 weeks after antibiotic cessation, CDI risk may persist up to 90 days after antibiotic cessation [16]. Methods for classifying pediatric $\mathrm{CDI}$, including type of diagnostic test utilized (e.g., toxin assay or nucleic acid amplification test), confirmation of symptomatology consistent with CDI (some studies use only laboratorybased results and may include asymptomatic children) and efforts to rule out more common causes of diarrhea, particularly among previously healthy children (e.g., viral gastroenteritis), also vary among studies. The performance of several C. difficile diagnostic tests is well described in adults [17], and compared with nucleic acid amplification tests (e.g., PCR), cytotoxin assays are less sensitive for identifying $C$. diffcile in stool. However, dedicated pediatric studies describing the performance of $C$. difficile diagnostic tests are few, and adult test performance data may not accurately translate to children. For example, a high proportion of false-positive cytotoxin assays in children has been reported [18], but this finding has not been reported in adults. With the majority of children's hospitals now utilizing nucleic acid amplification tests to diagnose CDI [19], and the unlikely clinical significance of detection of $C$. difficile by PCR in the absence of toxin detection [20], utilization of PCR in a population of patients with high prevalence of asymptomatic carriage of $C$. diffcile may bias results from pediatric CDI studies.

In summary, pediatric CDI is reported increasingly among children without hospital or antibiotic exposure. However, misclassification of colonized children as infected, or underreporting of antibiotic use, may bias these data. Future studies are needed to define $C$. difficile testing performance more precisely in pediatric patients and to establish a reliable and reproducible case definition for pediatric CDI, better differentiating colonized from infected patients. These definitions, particularly when utilized prospectively, will permit more reliable evaluation of risk factors, treatment efficacy and outcomes for pediatric CDI.

\section{Financial \& competing interests disclosure}

LK Kociolek receives grant support from Merck. DN Gerding is a board member for Sanofi Pasteur, Actellion, Merck and Rebiotix, and a consultant for Summit and Viropharma. DN Gerding is a patent holder for a product with Viropharma. The authors have no other relevant affiliations or financial involvement with any organization or entity with a financial interest in or financial conflict with the subject matter or materials discussed in the manuscript apart from those disclosed.

No writing assistance was utilized in the production of this manuscript.

\section{References}

1 Kelly CP, LaMont JT. Clostridium difficile - more difficult than ever. N. Engl. J. Med. 359(18), 1932-1940 (2008).

2 Freeman J, Bauer MP, Baines SD et al. The changing epidemiology of Clostridium difficile infections. Clin. Microbiol. Rev. 23(3), 529-549 (2010).
Tamma PD, Sandora TJ. Clostridium difficile infection in children: current state and unanswered questions. J. PediatricInfect. Dis. Soc. 1(3), 230-243 (2012).

4 Gerding DN. Clostridium difficile infection prevention: biotherapeutics, immunologics, and vaccines. Discov. Med. 13(68), 75-83 (2012).
5 The Centers for Disease Control and Prevention. Antibiotic resistance threats in the United States, 2013. www.cdc.gov/drugresistance/threatreport-2013

6 Dubberke ER, Gerding DN, Classen D et al. Strategies to prevent Clostridium difficile infections in acute care hospitals. Infect. 
Control Hosp. Epidemiol. 29(Suppl.1), S81-S92 (2008).

7 Benson L, Song X, Campos J, Singh N. Changing epidemiology of Clostridium difficile-associated disease in children. Infect. Control Hosp. Epidemiol. 28(11), 1233-1235 (2007).

8 Sandora TJ, Fung M, Flaherty K et al. Epidemiology and risk factors for Clostridium difficile infection in children. Pediatr. Infect. Dis. J. 30 (7), 580-584 (2011).

9 Kim J, Shaklee JF, Smathers S et al. Risk factors and outcomes associated with severe Clostridium difficile infection in children. Pediatr. Infect. Dis. J. 31(2), 134-138 (2012).

10 Tschudin-Sutter S, Tamma PD, Naegeli AN, Speck KA, Milstone AM, Perl TM.

Distinguishing community-associated from hospital-associated Clostridium difficile infections in children: implications for public health surveillance. Clin. Infect. Dis. 57(12), 1665-1672 (2013).

11 Wendt JM, Cohen JA, Mu Y et al. Clostridium diffcile infection among children across diverse US geographic locations. Pediatrics 133(4), 651-658 (2014).

12 Dumyati G, Stevens V, Hannett GE et al. Community-associated Clostridium difficile infections, Monroe County, New York, USA. Emerg. Infect. Dis. 18(3), 392-400 (2012).

13 Kelly CP, Kyne L. The host immune response to Clostridium difficile. J. Med. Microbiol. 60 (Pt 8), 1070-1079 (2011).

14 Schutze GE, Willoughby RE; Committee on Infectious Diseases; American Academy Of Pediatrics. Clostridium difficile infection in infants and children. Pediatrics 131(1), 196-200 (2013).

15 Kociolek LK, Shulman ST, Gerding DN. Laboratory diagnosis overestimates the burden of pediatric community-onset Clostridium difficile infection: impact of symptom review, culture, and typing on case validation. Presented at: ID Week, San Francisco, CA, USA, 2-6 October 2013 (Poster 1361).

16 Hensgens MP, Goorhuis A, Dekkers OM, Kuijper EJ. Time interval of increased risk for
Clostridium difficile infection after exposure to antibiotics. J. Antimicrob. Chemother. 67(3), 742-748 (2012).

17 Burnham CA, Carroll KC. Diagnosis of Clostridium diffcile infection: an ongoing conundrum for clinicians and for clinical laboratories. Clin. Microbiol. Rev. 26(3), 604-630 (2013).

18 Toltzis P, Nerandzic MM, Saade E et al. High proportion of false-positive Clostridium difficile enzyme immunoassays for toxin $\mathrm{A}$ and $\mathrm{B}$ in pediatric patients. Infect. Control Hosp. Epidemiol. 33(2), 175-179 (2012).

19 Kociolek LK, Sandora TJ. National variability in surveillance, testing, and infection prevention for Clostridium difficile infection in pediatric populations. Am. J. Infect. Control 41(10), 933-935 (2013).

20 Planche TD, Davies KA, Coen PG et al. Differences in outcome according to Clostridium difficile testing method: a prospective multicentre diagnostic validation study of $C$ difficile infection. Lancet Infect. Dis. 13(11), 936-945 (2013). 